\title{
Renewal Proposal
}

\section{Original Project Goals}

\section{PROJECT GOALS}

The long-term objective of this research is to develop a system for measuring and identifying a wide range of volatile organic hydrocarbons, including organochlorides, at ppb levels in-situ in the subsurface ("at-depth") using a fiber-optic REMPI probe. (In this renewal proposal we would also like to expand the range of analytes to include contaminated soil and certain metal pollutants such as $\mathrm{Hg}$ and $\mathrm{Pb}$ ). And, to do this in a ruggedized system that is compatible with existing fiber-optic sensors, Raman and fluorescence probes and image guides. The specific focus of much of the proposed work is to identify and optimize those experimental parameters which effect the in-situ determination of organic molecules using resonance-enhanced multi-photon ionization (REMPI). To accomplish this goal we will systematically investigate the dependence of REMPI on laser wavelength, power and other experimental parameters for a variety of high-priority groundwater and vadose zone contaminants to determine optimal measurement conditions. Emphasis will be placed on visible or fiber-optic compatible wavelengths of excitation so that the high transmission of fiberoptics can be fully utilized. A fiber-optic REMPI system is being designed that is suitable for integration into an existing cone penetrometer system being delivered by LLNL. Fiber-optic probe designs that we tested in the first studies will be refined by LLNL. Probe performance will be predicted using previously developed optical techniques ${ }^{42,43}$ and also using electrostatic field models. The specific studies that will be performed are described in the following sections.

This work is novel in several ways:

- $\quad$ visible or fiber-optic compatible wavelengths of excitation will be the focus of much of the work;

- the research team is highly multidisciplinary with groups from both academic and DOE labs (Angel and Morgan from USC's Departments of Chemistry \& Biochemistry, and Colston and Carter from LLNL); and

- the proposed work includes both fundamental (new measurement approach with wide ranging applications) and applied aspects (REMPI sensors will be delivered) as well as performance testing using previously designed soil columns.

In this work we will study the use of both low-order (e.g., 1+1, short wavelength) and high-order (e.g., 2+2, 2+1 visible-wavelength) REMPI excitation schemes, (described below), for some high priority volatile organic and VOC contaminants to learn the best way to measure these UV-absorbing molecules in the subsurface. This knowledge will be applied to optimization of REMPI for in-situ measurements using fiber optics. We will study the REMPI $1+1,2+1$ and $2+2$ signals for different sample matrices as a function of excitation wavelength across a broad spectrum. Also, we will investigate the best optical geometry for the fiber optic launch and collection probes to determine the effect on the signal-to-noise ratio (SNR) and how best to integrate REMPI into existing fiberoptic probes that are used for Raman and fluorescence measurements. A result of this study should be a determination of the optimal excitation and collection conditions and sampling methods for organic contaminants in different matrices, and an understanding of the strengths and limitations of using fiber optics for REMPI sampling.

A highly multidisciplinary group of scientists has been assembled for this project. Angel (analytical chemist with experience with sensors and probe designs), Morgan (analytical chemist with expertise in data analysis techniques including multivariate analysis), and Colston and Carter (biophysics, electrical engineering with expertise in OPO measurements, cone penetrometer instrumentation, and subsurface measurements using fiber optics and remote Raman imaging). All 
groups will be working on the same topics but in this project we combine fundamental research (at USC) with technology development (at LLNL). The highly multidisciplinary nature of this research team will improve our chances of successfully completing the project with the generation of technology that can be used by DOE in support of subsurface remediation efforts.

\section{Research Changes in Renewal Application}

The original long-term goals of this research project have not changed. Several tasks have been refined and/or extended based on knowledge gained as a result of our prior research. Prior research accomplishments are described in detail in the Prior Results Section. Research tasks that are still needed to move towards our long-term goals are described in detail in the Research Plan Section. Briefly, most of our original tasks were completed or significant progress was made. However, we underestimated the amount of effort that would be required to make detailed REMPI measurements of chlorinated compounds and interferences using both UV and visible wavelength lasers, to purchase and install the necessary laser systems, and to teach new graduate students to use them effectively. However, this has been a tremendous learning experience for the students and they are now generating high quality REMPI data. We would like to complete the detailed studies of mixtures and interferent compounds that were outlined previously. Also, we would like to expand our studies to include the measurement of $\mathrm{VOC}$, and $\mathrm{Hg}$ and $\mathrm{Pb}$ contaminants in soils. We did not forsee the possibility of such measurements in our original proposal but now realize it is a natural extension of our vapor-phase work. In preliminary experiments, described below, we have found that contaminated soil samples can be measured using REMPI following laser desorption by the same laser pulse that is used to make the REMPI measurement. This capability should greatly extend the range of contaminants that can be measured by REMPI to include soils contaminated with VOCs, and also nonvolatile substances like larger organic compounds and even heavy metals.

We are also expanding the data analysis section to include multivariate analysis, PLS and simplex optimization as an efficient method for determining the optimal laser wavelengths for REMPI measurements of samples containing mixtures of contaminants. For this purpose we will bring in an expert in the area of multivariate analysis, Professor Steve Morgan, for one year. Preliminary analysis of samples containing 3 and 4-component mixtures of BTEX compounds was successful.

We will purchase a low-cost, ruggedized OPO laser system $(\sim 20 \mathrm{~K})$ to evaluate new REMPI sensors using soil columns that were designed as part of the original proposed work. This will also be useful to gain experience for future field trials. Field trials are not included in the proposed budget for this fundamental research proposal. However, considerable interest has been shown by those involved in the planning of integrated field demos in our REMPI sensor. We expect to pursue funding in the very near future to participate in a field demonstration, and we feel the REMPI sensor could be field tested almost immediately for selcted contaminants.

As a result of our collaboration with LLNL we have found that electrostatic field modeling can be very useful in the design of our REMPI sensors. Thus, LLNL will bring in an expert in this area to help with sensor optimization. LLNL will also construct third generation sensors and deliver a cone penetrometer system and detailed plans to USC in preparation for future field trials.

Estimate of Anticipated Unexpended Funds: \$0.00

\section{SIGNIFICANCE OF PROJECT TO THE EM MISSION}

\section{Contributions to The DOE Missions:}

A stated mission of the DOE is to develop new types of sensors for rapid detection of chemical contaminants in the subsurface including those associated with TRU and mixed wastes with 
emphasis on field portable devices and development of new transduction and chemical recognition schemes. The proposed research specifically addresses these stated needs. A REMPI sensor is a novel solution to the detection of VOCs (volatile organochlorides), and organic and inorganic contaminated soil. At the end of this research project we expect to demonstrate a sophisticated new sensor technology for VOC detection.

The proposed research focuses specifically on development of a novel monitoring technology for rapid detection of non luminescent or weakly luminescent contaminants such as TCE, PCE, cisand trans-DCE, $\mathrm{CCl}_{4}$ and BTEX subsurface environments in the vadose zone, or in ground water. Improved technologies are required by DOE for characterization and monitoring for site clean-up and waste processing applications. Especially needed are field deployable methods and devices for realtime monitoring to reduce dependency on laboratory analyses which are costly and time consuming. Improved sensing capabilities are needed for on-site analyses to provide real-time analytical capabilities for screening level and/or decision-quality data. Matrices of interest to the DOE are soils (or other solids), slurries, and aqueous and non-aqueous solutions. In-situ methods have been demonstrated for identifying high concentrations of organic liquids (e.g., Raman spectroscopy) and low concentrations of a few types of organic molecules (e.g., UV fluorescence and DUVAS), as well as a few selected organic molecules (e.g., sensors) at low concentrations. However, currently there is no method for selectively measuring low levels of volatile organochlorides and BTEX compounds of the type that would be indicative of subsurface contamination in the vadose zone.

Volatile organic chemicals are found in almost every natural water source on earth. This is primarily due to the poor disposal practices of the past and accidental spills. For example, in 1988 it was estimated that 33.1 million pounds of benzene and 344.6 million pounds of toluene were released into the environment. ${ }^{14}$ Once these chemicals have been introduced into the ground, they begin to partition into the water supplies and collect in wells and other storage areas. Since clean-up of contaminated water supplies is very expensive, it is desirable to develop techniques that can be used on-site to rapidly identify the organic pollutant as well as the amount of contaminant present.

Gas chromatographs (GC) are the most commonly used instruments for the detection of trace volatile organic compounds in water samples. ${ }^{\mathbf{1 5 , 1 6}}$ For these analyses, there is typically a preconcentration step that extracts the sample from the water and requires a great deal of time and effort. This extraction can be through various methods such as activated charcoal, liquid/liquid extraction, or solid phase microextraction (SPME). ${ }^{17,18}$ Since both the pre-concentration and GC separation steps require time and sample preparation, it would be favorable to have a fast, remote analysis capability.

Volatile organochloride (VOC) contaminants and co-contaminants, as well as fuel hydrocarbons like BTEX (benzene, toluene, ethylbenzene and xylenes) are found at many industrial plants and federal sites. Many sites of the U. S. Department of Energy are contaminated with mixtures of VOCs including tetrachloroethylene (TCE), perchloroethylene (PCE), dichloroethylene (DCE) and carbon tetrachloride $\left(\mathrm{CCl}_{4}\right)$, and by BTEX. There has been a concern that TCE and PCE has contributed to the ozone depletion in the atmosphere. TCE is considered as an important environmental pollutant because laboratory bioassays have shown that its metabolized products can cause nephrotoxicity and nephrocarcinogenicity in laboratory animals. There is a strong need to develop a sensor that can detect liquid and vapor-phase VOCs and related chlorinated compounds in-situ.

Monitoring volatile organics and VOCs by discrete sampling is costly and time consuming. Insitu monitoring would reduce the labor-intensive process of sampling, handling, and shipping samples for analysis. In-situ monitoring would also aid in situations where monitoring site access is difficult and costly, or where conditions may pose safety hazards to samplers. In-situ sampling with real-time monitoring of contaminant concentrations may support the construction of fully automated 
treatment systems that could substantially reduce operating costs. At present, concentrations of volatile organics and VOCs are measured by discrete sampling and analysis in field laboratories. These methods require approximately 2-3 weeks for turn around. In general, laboratory analytical work is highly accurate, but time delays and high cost (e.g., $\$ 150 /$ sample or more) are considered to be significant drawbacks. In-situ monitoring would lower the analytical chemistry cost of vadose zone and groundwater measurements, pump and treat projects, on-line process measurements, and characterization by cone penetrometry. This research could lead to the development of a new generation of advanced monitoring technologies that can help address the DOE needs in remote chemical sensing of subsurface environments as well as in biomonitoring of health impacts associated with energy technology development.

Contaminated ground water, soils, and buried wastes are candidates for in-situ monitoring. This proposal addresses the need to measure volatile organics, especially VOCs and BTEX, in-situ or "at depth", in subsurface environments. Remote in-situ monitoring technologies could offer significant advantages, including: Important cost reduction of clean-up by eliminating or minimizing excavation, transportation, sample handling and disposal of wastes; Reduced health impacts on workers and the public by eliminating exposure to contaminants and wastes during monitoring; and Remote treatment and remediation of inaccessible sites such as deep subsurface contaminants.

In-situ sensing via fiber optics has great potential for reducing expense, speeding up site characterization, and increasing the sensitivity and accuracy with which measurements can be made. Unfortunately, VOCs and most other volatile organics have no easily measured optical signature at low concentrations, and optical sensors have only been successfully demonstrated for $\mathrm{CHCl}_{3}$ and $\mathrm{TCE}$ and these operate in a non-reversible manner. ${ }^{1}$ As a result, fiber-optic sensors have not been generally applicable to volatile organics and VOC measurements at ppb levels, and sensors that are currently available are not selective.

The development of higher-order REMPI excitation schemes is novel and has the potential for a "breakthrough" in the area of VOC contaminant measurements. If successful this will allow $\underline{\text { in-situ }}$ measurement and identification of trace levels of difficult to measure species such as TCE, PCE, DCE, $\mathrm{CCl}_{4}$ and BTEX compounds that is not possible using any existing method. The approach is also general enough that it can be applied to inorganics, including heavy metals.

\section{Scientific Significance And Rationale}

Although the monitoring technologies developed in this project have a wide range of applications, a few examples of specific applications are selected here to illustrate the scientific significance and rationale of the proposed approach.

Remote Chemical Sensors. The development of remote chemical monitoring technologies in this project are important for several reasons: 1) they are cost-effective as a result of their in-situ, rapid chemical sensing capabilities, (2) they can probe hard-to-reach regions such as subsurface environments, and (3) they decrease the health impacts on workers and the public by reducing exposure during monitoring operations.

REMPI Probes. Luminescence techniques are very well known for their sensitivity to detect certain compounds. However, this is not the case for non luminescent or weakly luminescent contaminants. Important examples include TCE, PCE, DCE and related chlorinated volatile organic compounds. TCE and PCE have been found in many DOE hazardous waste sites and in underground environments. Conventional luminescence techniques cannot be used because TCE and PCE are nonluminescent. Also, even though BTEX compounds are luminescent when excited at UV wavelengths, the luminescence spectra are not useful for "fingerprinting," these compounds being very broad and 
overlapping. Also, the absorption bands are broad making selective excitation impossible. In addition, short UV wavelengths aren't very compatible with fiber optics. We have shown that visible wavelength excited REMPI can be extremely sensitive and selective and it does not suffer from either of these problems to the same extent as fluorescnce or absorption.

New Family of Optical Sensors. This research will ultimately result in an entirely new type of optical chemical sensor resulting in many different types of sensing opportunities including organic vapors, dissolved species, soil contaminants and even heavy metal contaminants like $\mathrm{Hg}$ and $\mathrm{Pb}$.

\section{REMPI Background}

\section{BACKGROUND}

Resonance enhanced multiphoton ionization (REMPI) is a useful technique for obtaining both qualitative and quantitative information about volatile organic pollutants. It has been used extensively as a selective ionization source for mass spectrometers ${ }^{\mathbf{6 - 1 2}}$ as well as in spectroscopic combustion analysis. ${ }^{\text {13-16 }}$ In the REMPI technique, a tunable pulsed laser is used to electronically excite a specific molecule using one or more photons. A $1+1$ excitation scheme refers to a twophoton process where one photon creates the excited molecule and another photon ionizes the molecule from the excited state. In a $2+2$ excitation scheme two photons are used to excite then ioinize the molecule-a four-photon process. As shown in Fig. 1 the main difference between resonance ionization and laser ionization (LI) is that an electronic excited state is involved in REMPI. This not only results in very large enhancements but is also the reason REMPI can be used as a qualitative technique. While excited, the subsequent absorption of an additional photon(s) then ionizes the molecule. Ionization probability is much larger for REMPI than for LI because the "real" state in REMPI is much longer lived than the "virtual" state in LI. Also, because of the large enanecements in REMPI, even four-photon excitation schemes provide very high sensitivity. And, it is important to point out that REMPI requires low to moderate laser pulse energy.

Once ionized, an electrode that is biased at a high positive or negative potential is used for efficient electron or ion collection. The current created by these electrons/ions flowing to the electrode is then measured as a voltage drop across a "leak resistor," and the resulting voltage is therefore proportional to the concentration of the specific species being ionized. Due to the nonlinear nature of this technique it is important to have high power densities, small focused beam, near the collection electrode. A higher power density increases the probability of ionizing the specific molecule of interest. Although the electrode is typically biased at several hundred volts, the current is extremely low requiring only a very small relatively well-regulated power supply.

In the REMPI technique a relatively low power pulsed laser is focused to a small spot to induce molecular ionization in a multiphoton, $\mathbf{N}$-photon, process where the multiphoton energy exceeds the ionization potential. In nonresonant multi-photon ionization (MPI), the ionization cross section, $\mathrm{d}_{\mathrm{N}}$, is low but increases with increasing photon flux, $\mathrm{I}$, with $\mathrm{d}_{\mathrm{N}}$ being proportional to $\mathrm{I}^{\mathrm{N}}$. By tuning the laser so that the energy sum of the $\mathbf{N}$ photons is in resonance with a real intermediate resonance state, $E_{f}$, (REMPI) the ionization cross section is enhanced by up to $10^{6}$ if the $E_{o} \rightarrow E_{f}$ transition is an allowed transition of the molecule $\left(E_{o}\right.$ is the ground state, $E_{f}$ is the energy of the resonant electronic state). This is shown schematically in Fig. 1. The greatly enhanced ionization cross section in REMPI results in extremely high ion yields and thus very high sensitivity. Because ions can be collected very efficiently this technique is one of the most sensitive in-situ methods for applications such as combustion diagnostics. ${ }^{\text {23-25 }}$ REMPI has sensitivity comparable to fluorescence but can be used to measure non-fluorescent compounds. However, it is more selective than 
fluorescence and has been used to measure trace levels of molecules in complex matrices such as flames and plasmas.

Fig. 1 shows several possible excitation schemes for REMPI. We have found the $(1+1)$ and $(2+2)$ schemes to be very useful for BTEX compounds, giving ppb detection limits using $~ 4-6 \mathrm{~mJ} / \mathrm{pulse}$. We have used the $(2+1)$ scheme for chlorinated compounds including DCE, PCE and TCE. In recent studies we have found that this scheme can be used to selectively measure cis-DCE and trans-DCE and easily determine the relative amount of each isomer even when both are contained in a mixture with other chlorinated compounds such as TCE and PCE. This is an exciting result considering the relative importance of the isomers of DCE. Fig. 2 shows REMPI excitation scans over a relatively narrow wavelength range for cis-DCE (lower spectrum), trans-DCE (middle spectrum) and a mixture containing both isomers (top spectrum). The two DCE isomers are completely resolved in these relatively lowresolution REMPI spectra.

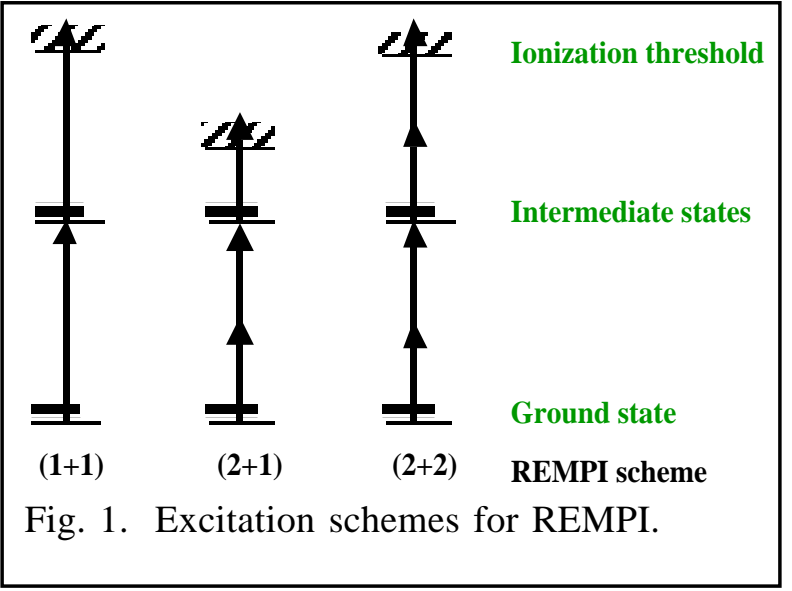

REMPI is widely used to obtain multiphoton spectra because of its high sensitivity and simplicity. Although there have been a few reports of its analytical use most reports have focused on studying the resonance enhanced multiphoton ionization process itself rather than its use for quantifying chemical species. However, applications of this technique are beginning to appear. For example the spectral sensitivity and selectivity of resonance-enhanced multi-photon ionization spectroscopy for detecting various chloroethylenes has been demonstrated and REMPI has been used to measure many radical species including $\mathrm{O}, \mathrm{H}, \mathrm{OH}, \mathrm{CH}, \mathrm{CO}, \mathrm{HCO}, \mathrm{CH}_{3}$ and chlorinated flame radicals. ${ }^{23-31}$ REMPI has been used most widely for measuring gas-phase spectra of simple molecules, molecule fragments and radical species. However, as indicated above recent studies have used REMPI for organic molecules, and we recently demonstrated its use for measuring volatile organic molecules in-situ.

Due to its sensitivity and qualitative capabilities, REMPI, is an excellent technique for remote analysis of many types of volatile organics in the environment. A REMPI probe is made by simply placing a small biased electrode near the focus of this laser pulse, making it possible to make a simple and remote probe that can be placed down a well or other inaccessible area for remote trace analysis. Most organic contaminants absorb most strongly in the UV. For example, BTEX compounds (e.g., benzene, toluene, ethylbenzene and xylenes) absorb strongly in the 240 to $280 \mathrm{~nm}$ range. In previous work we used $266 \mathrm{~nm}$ excitation in a $1+1$ excitation scheme to measure toluene at $\sim 1$ ppb with a fiber-optic probe. However, in that

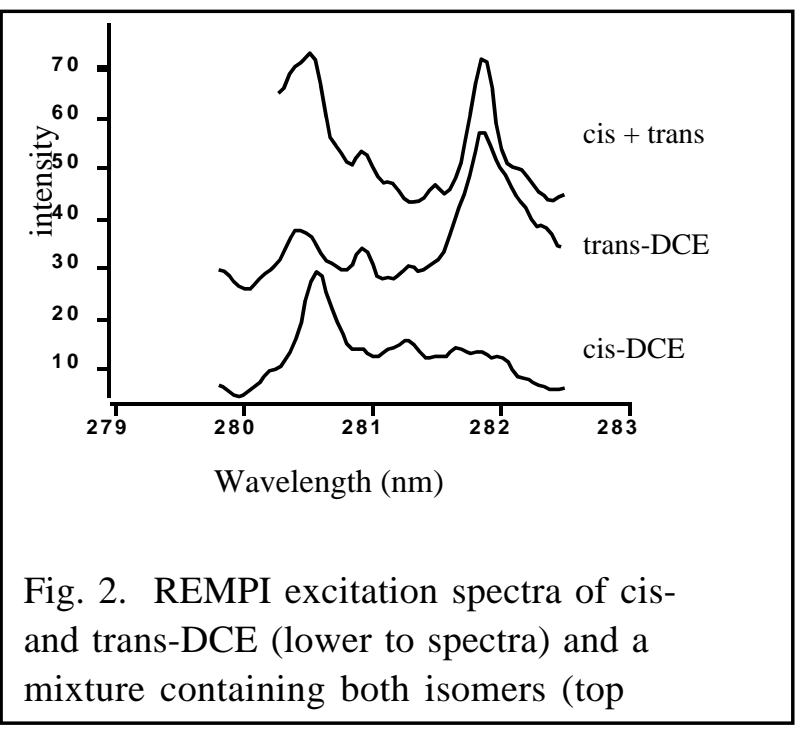
work we also showed that four-photon REMPI, in a $2+2$ excitation scheme at $532 \mathrm{~nm}$, could be used 
to measure toluene at concentrations $<8 \mathrm{ppb}$. This result was achieved using a relatively small laser with 4-6 mJ per pulse. This is an important result because it demonstrates that REMPI can be used to measure low levels of organic contaminants with a small visible-wavelength laser, and at wavelengths that are compatable with fiber-optic probes.

Before REMPI can be applied to the measurement of subsurface contaminants it is necessary to make fundamental measurements to characterize it for this purpose, especially comparing $1+1$ and $2+2$ excitation schemes. And, the optimal laser wavelength for the analytes of interest. The optimal wavelength for a REMPI measurement does not always correspond to the maximum sample absorbance, because of lower background ionization in regions of lower absorbance, ${ }^{\mathbf{3 3}}$ or rapid relaxation to spin-forbidden states ${ }^{2}$. Although these results were obtained at low pressure and the experiments aren't too related, it is not known if similar results, especially background ionization, might be observed in one-atmosphere experiments. In this application systematic studies of laser wavelength have rarely been done. Most detailed excitation wavelength studies have been carried out for flame species. ${ }^{1922}$ Thus, an important part of this work is to study the excitation wavelength dependence of the REMPI signal for the contaminants of interest. We also need to determine what other experimental parameters can be optimized for an analytical measurement, and to evaluate potential interferences. The excitation wavelength dependence of REMPI signals will be determined for several high-priority pollutants using $1+1,2+1$ and $2+2$ and 2-color excitation schemes. REMPI signals as a function of sample matrix, sample state (dry, wet, etc.), and excitation wavelength have not been obtained in a systematic way for the compounds of interest and such studies are crucial to making this a reproducible analytical technique. No fiber-optic REMPI studies other than our own have appeared in the peer-reviewed literature.

We have investigated the use of fiber optics to deliver the laser to the sample and to collect the resulting ion emission using a small electrode surrounding the fiber. Using this probe we achieve $\sim 1$ $\mathrm{ppb}$ detection limits for BTEX compunds using only a few $\mathrm{mJ} /$ pulse of laser excitation. The use of fiber optics for REMPI will minimize the need for grab-sampling. In prior work we began studies of the REMPI excitation wavelength dependence at UV and visible wavelengths for sample mixtures. A result of further study should be a determination of the optimal excitation wavelengths and sampling conditions for organic contaminants in different matrices, and an understanding of the strengths and limitations of using fiber optics for REMPI. Our preliminary REMPI work is quite exciting. ${ }^{12,13} \mathrm{~A}$ 90-s analysis was shown to be sufficient to identify and quantify ppb levels of toluene, even in a complicated sample like gasoline, and with little or no interference from benzene. The laboratorybuilt fiber-optic REMPI probe is small and rugged enough for field work.

\section{SUMMARY OF RESULTS FROM PREVIOUS RESEARCH}

In the original proposal the following specific studies were described to move towards our long-term goals.

1) Obtain gas-phase absorption spectra in dry and saturated water vapor conditions.

2) Measure REMPI excitation profiles in the UV (1+1) and visible $(2+2)$ for selected high priority organic contaminants, and determine the optimal wavelength for each compound.

3) Compare UV (1+1) and visible $(2+1$ and 2+2) excitation in terms of analytical figures of merit, such as sensitivity, detection limits, selectivity, and interference effects for the same compounds.

4) Test the REMPI probe in a soil column that mimics subsurface conditions.

5) Determine the optimal laser source for use in making field measurements of these compounds. This is a trade-off between detection limit, price and ruggedness of the laser system.

6) Design a fiber-optic REMPI probe that is compatible with an existing LLNL cone penetrometer 
Raman probe and include an image guide for direct visualization of the sampling region.

\section{The following list is a summary of the most important accomplishments from our prior research.}

- $\quad$ Selective measurements of cis- and trans-DCE were demonstrated even in the presence of TCE and PCE.

- $\quad$ REMPI spectra of 3 and 4-component mixtures of BTEX compounds were measured. Optimal sets of excitation wavelengths were determined using multivariate analysis.

- Demonstrated 1-2 ppb detection of BTEX cpds. using a non-lensed fiber-optic REMPI probe.

- Developed a very simple non-lensed fiber-optic REMPI probe and demonstrated detection limits and dynamic range comparable to a lensed probe.

- Optimized the fiber-optic probe in terms of fiber diameter, numerical aperture and electrode thickness. Optical modeling and electrostatic field modeling was begun.

- Demonstrated REMPI measurements of soil samples using laser desorption, where the same laser pulse was used for both desorption and sample ionization.

- A soil test column was designed and a measurement protocol was developed for sensor tests.

- Image guides were shown to be useful for in-situ imaging and spectroscopy. We also jointly published with LLNL several papers describing the use of image guides for Raman spectroscopy.

- Numerous papers were presented including a paper describing the new probe with an electrostatic field model, and an invited paper at RIS 2000, the 10th International Symposium on Resonance Ionization Spectroscopy and Its Applications, in Knoxville, Tennessee. Some of these papers are listed in the Collaborative Work Section of this renewal proposal.

Of the studies described in the original proposal we made significant progress or completed 1, 2, 3, 5 and 6. Study 4 was not completed although an appropriate soil column was designed by Dr. Gribb after leaving USC. In this renewal proposal we would like to complete Study 4 using the non-lensed fiber-optic probe, and complete the other studies as we move towards our long-range goals.

\section{Progress on the Original Proposed Studies}

Study 1: Complete.

Study 2: Significant progress was made and detailed excitation REMPI profiles were obtained at UV wavelengths for the BTEX compounds. Detection limits were determined and optimal excitation wavelengths were determined for each contaminant. Preliminary analysis to determine optimal visible excitation wavelengths for 3-4 component mixtures was begun. We have shown that all of these compounds can be selectively measured in the UV using REMPI at atmospheric pressure without a buffer gas. However, this task needs to continue for visible wavelengths using more sophisticated forms of multivariate data analysis as described below. The best excitation scheme seems to be the use of two wavelengths, one on and one off an absorption band. A buffer gas also seems to provide a sensitivity advantage.

Excitation REMPI profiles have been made using $2+1$ excitation for TCE, PCE, cis- and transDCE over selected wavelength regions. However, we underestimated the amount of effort involved in making detailed visible measurements of mixtures and interferences. Further work is needed of the basic spectroscopy of these compounds before we can determine the optimal wavelengths for each, especially in mixtures. Measurements in aqueous solution have also not been completed. 
Study 3: Progress has been made on Study 3. We compared detection limits and sensitivity for BTEX compounds using UV excitation wavelengths and determined optimal excitation wavelengths for single components. However, detection limit and sensitivity studies using visible wavelengths were only partially completed because of problems with the OPO laser system at LLNL. We have moved this study to USC using a tunable dye laser system that we are upgrading for this work. Interference effects were begun but not completed. We found that common solvents such as alcohols did not interfere with our measurements of BTEX or VOC compounds. Preliminary analysis of mixture using multivariate analysis looks promising. We would like to continue this line of research and have added an investigator with expertise in the area of multivariate analysis.

Study 4: A soil column and measurement protocol was developed by Dr. Gribb. We will construct this column and use it to test potential interferences. These tests will mimic the conditions of real soil systems. A small, ruggedized OPO laser system will be purchased to support this effort.

Study 5: This study is changing as we learn more about the spectroscopy of these compounds. A low-cost, ruggedized OPO laser system $(\sim 20 \mathrm{~K})$ seems best at this time for measuring BTEX compounds and should give ppb detection limits at visible wavelengths. In the case of chlorinated compounds the detailed spectroscopic mixture studies need to be completed. The best way to make measurements seems to be the use of a dual-wavelength differential excitation schemes.

Study 6: This study is complete but we will develop a third generation probe based on prior results with the non-lensed probe. We developed a unique single-fiber, non-lensed REMPI probe that has excellent signal to background and gives ppb detection limits for BTEX compounds (see picture in Fig. 3). However, optimization studies indicate that this design can be further refined (see description of probe studies below). In the renewal proposal we will refine this design and test it in soil columns. It has not been tested for measuring VOCs.

The new probe contains no lenses and no optical elements other than the optical fiber. Thus, it is self-aligning and extremely rugged and reliable. It is also very sensitive as shown by comparisons to a lensed probe in Fig. 4. Fig. 4 shows calibration curves for benzene using the lensed (left) and nonlensed (right) probe with $532 \mathrm{~nm}$ excitation ( 1 ppb was measured using $266 \mathrm{~nm}$ ). Although we have not yet tested this probe for chlorinated compounds the performance is expected to be similar, dependent mainly on the excitation wavelength.

Expanded Tasks: On preliminary experiments using soil samples we found that the laser pulse that is used for REMPI excitation is

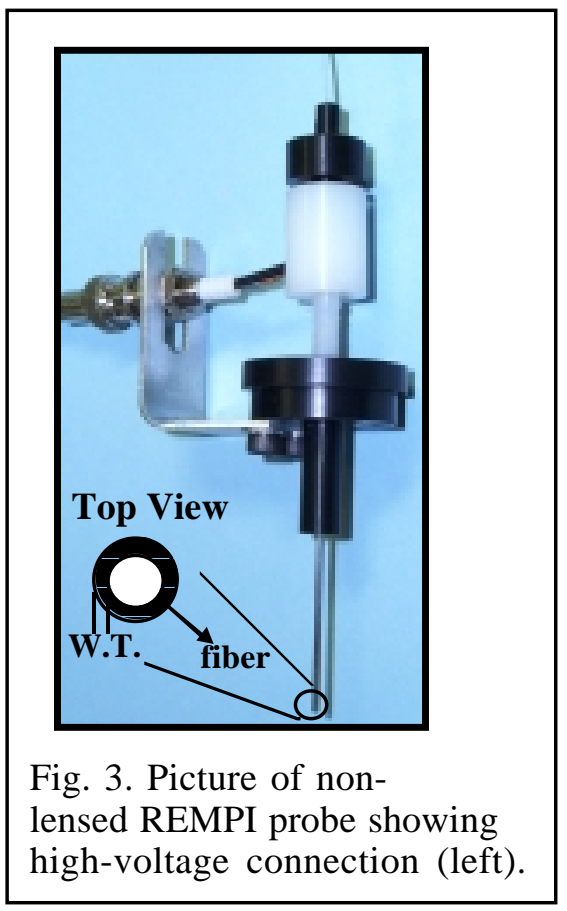
also effective at desorbing organic vapors. Fig. 5 shows a schematic of this experiment. In preliminary measurements the soil sample was placed below the REMPI electrode in such a way that a single laser pulse could be used both to desorb the VOC from the sample and to ionize the desorbed VOC vapor. As shown in Fig. 6 the REMPI signal following laser desorption is very large. This technique allows REMPI measurements to be made on contaminated soil samples even for non- 
volatile compounds. Fig. 7 shows the development of the REMPI signal as a function of time after the laser pulse for a toluene spiked soil sample. The signal develops almost immediately following the laser pulse and reaches a value that is greater than that achieved by allowing the sample to sit at equilibrium in the closed container.

We would like to expand our tasks to include a study of laser desorption REMPI for contaminated soil samples. We will develop this system using a low power desorption laser focused onto the surface of the soil. A REMPI excitation pulse will be brought in parallel to the sample surface. We will compare the use of a low-cost Nd:YAG laser and a pulsed diode laser for sample desorption. This is described in detail in the Experimental Methods section.

\section{Relation of Prior Results to Proposed Activities in the Renewal Period}

Although we made significant progress in all areas of the original proposal we have not achieved all of our long-term goals. A significant effort is still needed to complete the UV and visible wavelength REMPI excitation studies and interference studies, especially for VOC mixtures.

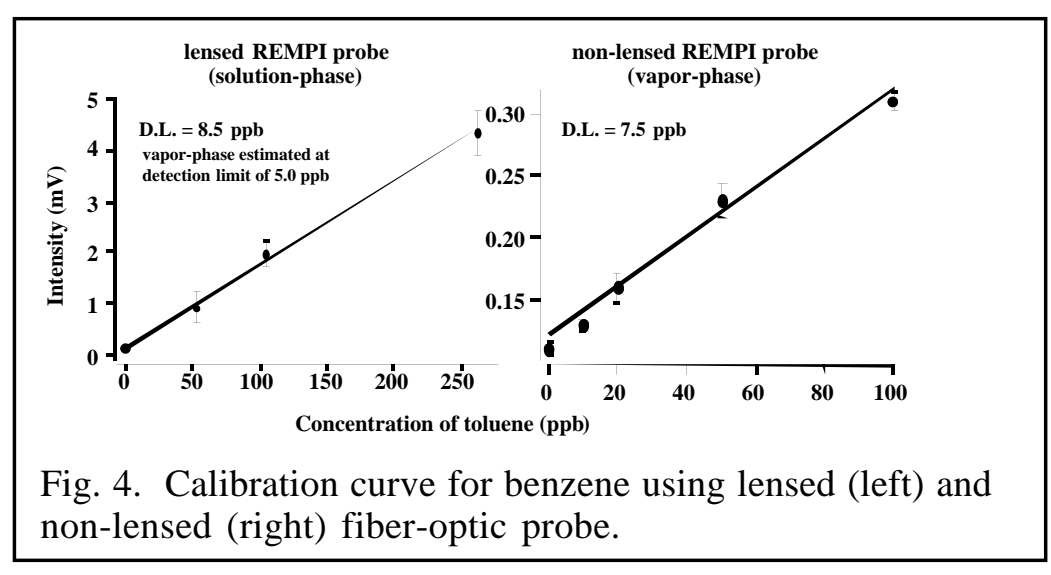
Much of the first three years of this study involved: improving and characterizing the fiber-optic probe measurement techniques; determining detection limits and sensitivity for individual BTEX compounds; determining optimum excitation wavelengths for these compounds using UV and visible wavelengths; setting up a high resolution REMPI system using a tunable dye laser; and measuring REMPI excitation spectra of chlorinated compounds. To achieve our long-range goals will require much more detailed spectroscopic characterization of mixtures of the chlorinated compounds. We underestimated the amount of time and effort this task would take in our original proposal, especially the mixture studies and determination of optimal excitation schemes. The soil column studies were not completed as a result of Dr. Gribb leaving USC. We feel those studies should be completed before field tests are considered.
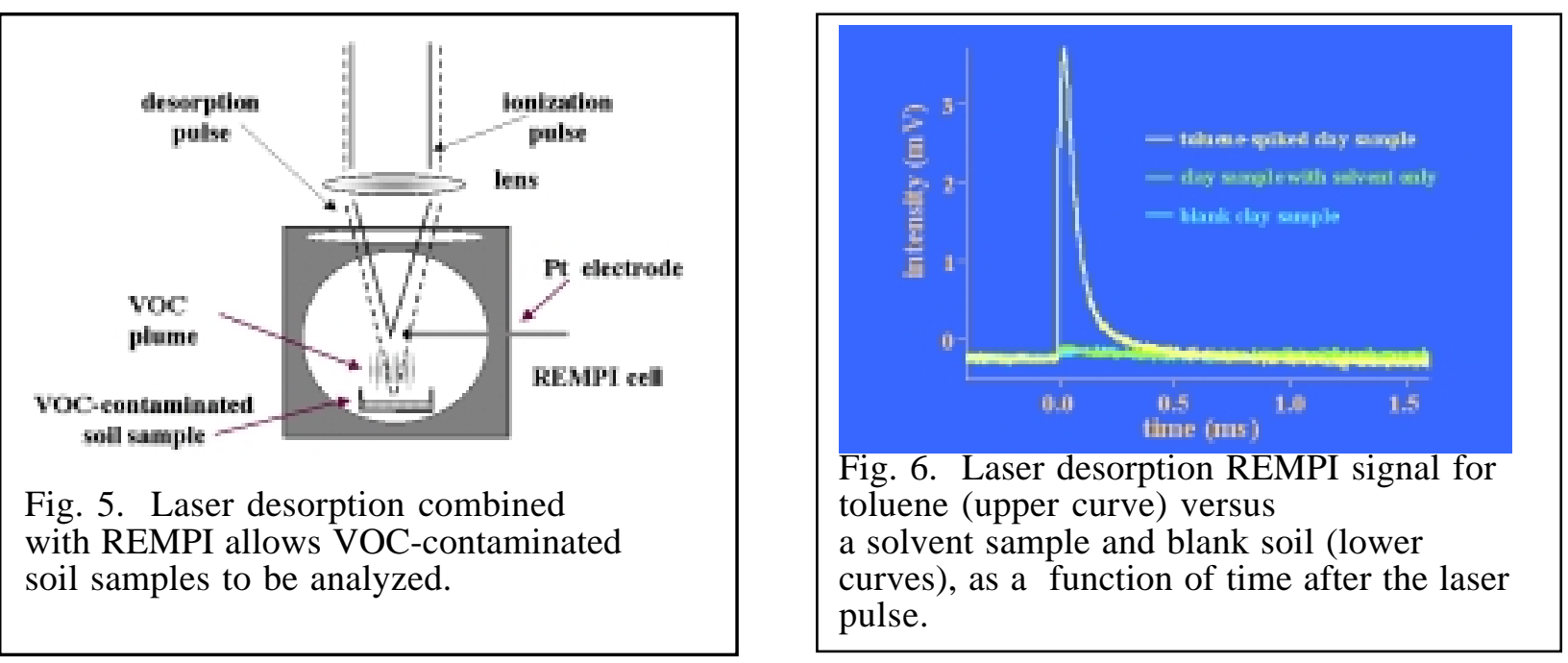


\section{Technical Approach}

\section{RESEARCH PLAN}

Resonance Enhanced Multiphoton Ionization measurements are relatively simple requiring only a relatively low-energy pulsed laser and a simple biased electrode to measure the ionization current. Resonant excitation provides extremely large enhancement and discrimination over non-resonant laser ionization. REMPI is extremely sensitive at atmospheric pressure, comparable to fluorescence, and provides ppb level detection. In some cases the addition of a small amount of argon gas is added to increase the REMPI signal. What makes the technique particularly attractive for subsurface monitoring is that high selectivity can also be achieved by taking advantage of the absorption spectrum of the molecule. Resonance enhancement is only observed with excitation into the molecules absorption bands so the absorption spectrum can be obtained directly or a two-wavelength measurement can be made. A molecular "fingerprint" can even be measured in a complex mixture because the REMPI excitation bands are relatively sharp and non-resonance ionization signal is low. Also, only optical access is needed for ionizing the molecule, with current measured by an external electrode that surrounds the tip of the optical fiber. The composition of the sample is accomplished by measuring the excitation REMPI spectrum or characteristic wavelengths for the molecules in the sample. There are reports of the use of REMPI for measuring and identifying molecular species either directly by the excitation spectrum or by the use of proportional counters. ${ }^{2-9}$

In the proposed work we will study the use of both low-order (e.g., 1+1, short wavelength) and high-order (e.g., 2+1 and 2+2 visible-wavelength) REMPI excitation schemes, (described below), for some high priority volatile organic and VOC contaminants to learn the best way to measure these UV-absorbing molecules in the subsurface. This knowledge will be applied to optimization of REMPI for in-situ measurements using fiber optics. Also, we will investigate the best optical geometry for the fiber optic launch and

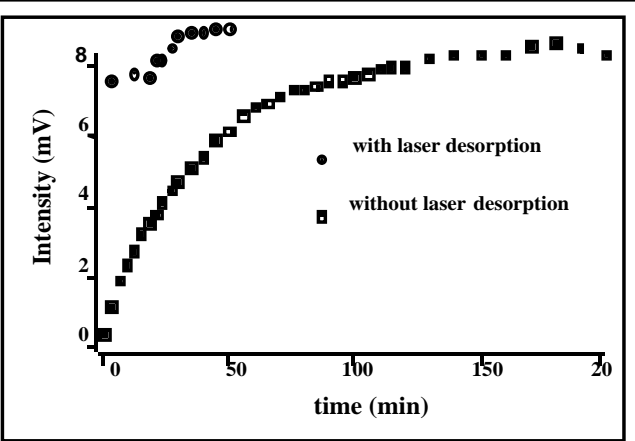

Fig. 7. Development of REMPI toluene signal for a clay sample with (top curve) and without (lower curve) laser desorption. collection probes to determine the effect on the signal-to-noise ratio (SNR) and how best to integrate REMPI into existing fiber-optic sensor platforms and probes that are used for Raman and fluorescence measurements. Finally, we will study the REMPI $1+1,2+1$ and $2+2$ signals for different sample matrices as a function of excitation wavelength across a broad spectrum. A result of this study should be a determination of the optimal excitation and collection conditions and sampling methods for organic contaminants in different matrices, and an understanding of the strengths and limitations of using fiber optics for REMPI sampling. Multivariate analysis will be used to determine the optimal wavelengths for determination of mixtures.

\section{RESEARCH DESIGN AND METHODOLOGIES}

REMPI System We now have two different REMPI systems at USC. One system uses a low-cost Nd:YAG laser and a non-lensed fiber-optic REMPI probe. This system has been described in several papers (see Collaborative Arrangements Section). The other system uses a high-resolution tunable pulsed dye laser as the excitation source and a sealed stainless steel REMPI cell. 


\section{Specific new studies to be carried out.}

1. Continue to measure REMPI excitation profiles in the UV $(1+1)$ and visible $(2+1,2+2)$ for selected high priority organic contaminants, and determine the optimal wavelength for each compound both alone and in mixtures of other compounds. This will include measurements in the presence of potential interferences such as common solvents and water vapor. (USC)

2. Compare UV $(1+1)$ and visible $(2+2)$ excitation using the previously developed non-lensed fiberoptic REMPI sensor in terms of analytical figures of merit, such as sensitivity, detection limits, selectivity, and interference effects for the same compounds as in 1. (USC)

3. Multivariate analysis will be used to determine optimal excitation wavelengths for BTEX and organochloride compounds (USC).

4. Construct a soil column like that designed in the prior studies (LLNL). Evaluate the non-lensed REMPI probe in the soil column to simulate field conditions. These experiments are described in detail below.

5. Refine the fiber-optic REMPI sensor design with the help of electrostatic field modeling and build prototype devices for characterization (LLNL). Design new sensors around existing cone penetrometer and Raman probe designs (LLNL). Deliver a cone penetrometer to USC (LLNL). 6. Set up a laser desorption REMPI system using a pulsed diode laser for desorption. Compare a diode laser and low-cost Nd:YAG laser for sample desorption (USC). Determine the feasibility of using this system for measuring low-volatility samples including $\mathrm{Hg}$ and $\mathrm{Pb}$.

7. Investigate the use of a buffer gas (Ar) in the probe and ways to reduce the probe pressure. This study is described below. (USC)

8. Determine the easibility of measuring $\mathrm{Hg}$ and $\mathrm{Pb}$ in soil samples using laser desorption. (USC)

\section{Approach/Experimental Design}

Fiber launch conditions: One difficulty in using fiber optics for REMPI measurements is avoiding damage to the excitation fiber. Focusing the laser on the tip of the fiber can chip the face, or in some cases completely destroy the fiber's tip. In addition, it was also found that if too much power was launched into the fiber or into the cladding of the fiber, catastrophic damage to the core/cladding interface was observed. Since REMPI is a non-linear process it is important to launch as much power down the fiber as possible. By using a pinhole at the fiber tip, and placing the fiber just beyond the focus of the laser, it was found that optical fibers can withstand approximately $200 \mathrm{~mJ} / \mathrm{pulse}$ at 532, and this launch worked very well for REMPI experiments. ${ }^{\mathbf{3 2}, 37}$

Power density is crucial to obtaining quality REMPI signals and thus high sensitivity. It is important both to transmit the maximum amount of excitation light through the fiber, as well as to have a small spot size near the electrode tip. The power density near the electrode depends on the diameter and type of excitation fiber used. We find that small-diameter fibers using about 4-6 $\mathrm{mJ} /$ pulse gives the best result using the current non-lensed probe. Prior optimization studies of probe optics and the electrode geometry suggests that the performance can be significantly improved.

\section{Experimental Design}

Task 1. Measure REMPI excitation profiles in the UV $(1+1)$ and visible $(2+2)$ for selected high priority organic contaminants, and determine the optimal wavelength for each compound. Excitation spectra show that the optimum excitation wavelength for BTEX compounds is between 230 to $280 \mathrm{~nm}$ for $1+1$ excitation and about 460 to $560 \mathrm{~nm}$ for $2+2$ excitation. For the chlorinated 
compounds the wavelength range is 270 to $290 \mathrm{~nm}$ for $2+1$ excitation. Absorption spectra indicate a wavelength range of 190 to $250 \mathrm{~nm}$ for $1+1$ excitation. We are also investigating other excitation schemes including 2-color excitation for enhanced selectivity.

We will compare UV (1+1) and visible $(2+2)$ excitation in terms of analytical figures of merit, such as sensitivity, detection limits, selectivity, and interference effects for the same compounds. Sensitivity and detection limit studies will be carried out like those above but will be done using a number of different concentrations of each compound over a 3 to 4 magnitude range, to determine the linear/dynamic range, with the lowest concentrations being at or near the limit of detection. Five measurements will be made at each concentration for statistical analysis in order to calculate standard deviations. Sensitivity will be reported as the slope of the analytical calibration curve. Detection limit will be reported as three times the standard deviation of the background divided by the calibration slope ("3 sigma DL"). These measurements will be performed with and without the fiber optic probe. These determinations will be made at 3 different wavelengths each in the UV and visible range. This will allow us to more accurately determine the affect of the fiber on these measurements. Table 1 shows the measurement matrix for this study. The total number of measurements is very large, but necessary, and is one reason we did not complete this study in the first funding period.

Table 1. Test matrix for sensitivity and detection limit determinations.

\begin{tabular}{llll}
\hline Exp. No. & Chemical & Concentration ppb & Wavelengths \\
\hline \hline $1-30$ & TCE & $1,10,50,100,1000$ & 6 \\
$31-60$ & PCE & $1,10,50,100,1000$ & 6 \\
$61-90$ & DCE & $1,10,50,100,1000$ & 6 \\
$91-120$ & benzene & $1,10,50,100,1000$ & 6 \\
$121-210$ & xylenes & $1,10,50,100,1000$ & 6 \\
$211-240$ & toluene & $1,10,50,100,1000$ & 6 \\
\hline
\end{tabular}

Task 2. Selectivity and interference tests will be determined by measuring 2, 3, 4 and 5 component mixtures, dry vapor and above aqueous-phase solutions, of both BTEX, and chlorinated compounds (TCE, PCE and DCE). Interference by co-existing compounds during the analysis of a target compound would be expected to show up as a non-specific ionization current, that is dependent on the concentrations of interfering species. This will be studied by measuring the ion current for a fixed concentration of analyte, as non-target analyte concentrations are increased. A determination of the optimal wavelength for determination of a target compound in a mixture will be one result of this study. Table 2 shows the measurement matrix for this study. The number of measurements is large but necessary for continued progress on this project and for future field trials.

Task 3. Multivariate calibration and selection of optimal wavelengths for REMPI analysis.

One of the most widely used multivariate techniques in chemometrics is partial least squares regression (PLS). ${ }^{45-48}$ PLS defines latent variables (linear combinations of the original wavelengths) that best describe variations to be modeled in the data set (e.g., spectral variations related to concentration changes). The optimum number of latent vectors to adequately account for these variations is determined by iteratively fitting the model with increasingly more latent vectors and looking for a minimum in a plot of cross-validated error of prediction against the number of latent variables. Typically, calibration spectra are taken using a set of designed experiments that vary the 
concentrations of all analytes of interest. The variations in the spectra are modeled to relate the variations across the entire spectrum to the concentration of the components. Model validation is often conducted by cross-validation, which involves omitting spectra from the data set one at a time (or in some other systematic manner), and fitting the model to the remaining data. The spectrum that is left out is then fed to the model to predict its concentration and this process is repeated for every spectrum in the data set. The mean square error of prediction for all "left out" spectra is then used as a measure of model robustness and quality. External validation with a separate test data set, separate from the training set, is also recommended. Multivariate PLS models are often more robust than models based on single or selected groups of wavelengths because interferences and other variations in the spectral data are be explicitly modeled.

Table 2. Test matrix for interference determinations.

\begin{tabular}{lllll}
\hline Exp. No. & Target & \# Interferences & Condition & Concentrations \\
\hline \hline $1-20$ & TCE & B, X, T, PCE, DCE & dry, and wet & 2 \\
$21-40$ & PCE & B, X, T, TCE, DCE & dry, and wet & 2 \\
$41-60$ & DCE & B, X, T, PCE, TCE & dry, and wet & 2 \\
$61-80$ & benzene & B, X, T & dry, and wet & 2 \\
$81-140$ & xylenes & B, X, T & dry, and wet & 2 \\
$141-160$ & toluene & B, X, T & dry, and wet & 2 \\
\hline
\end{tabular}

We have performed designed experiments varying the relative amounts of BTEX components in 3- and 4-component mixtures. The complete set of REMPI 2+2 excitation spectra data from each set of experiments were modeled using PLS. A major objective was to identify wavelengths most informative for predicting concentration of BTEX compounds. The four-component mixture design discussed here involved spectra of single-component benzene, toluene, ethylbenzene, and $m$-xylene, along with various mixtures of all four components. The design points take the geometric shape of a three-dimensional simplex or tetrahedron (Fig. 8) and were generated using Design Expert (Stat-Ease Corp.). The single components are at the extreme vertexes and quaternary mixtures are located in the simplex interior. Each component spectrum was recorded twice and five additional replicate mixture spectra were included in the design of 20 experiments. ${ }^{49}$ Data analysis was performed using programs written in Matlab (The Mathworks, Inc.). Partial least squares was performed using the SIMPLS algorithm in the commercially available PLS Toolbox (Eigenvector Research, Inc.). PLS has become established to the extent that several other commercial programs for PLS are also available (e.g., JMP from the SAS Institute, and Grams 32 from Galactic Industries). Cross validation (Fig. 9) found 7 latent vectors were adequate to represent the combined concentration and spectral variation for all 4 analytes.

Single-component spectra of benzene and ethylbenzene are shown in Fig. 10A and 10B. PLS calibration plots for all four components had coefficients of determination $\left(R^{2}\right)$ of 0.99 or higher; plots for benzene and ethylbenzene are shown in Fig.s 10C and 10D. Plotting the regression coefficients against the wavelength variables enables identification of the optimal excitation wavelengths for each analyte. Note: the optimal wavelengths for each analyte in a mixture may not be the same as the optimal wavelengths for a single component. Wavelengths found to most informative for detection of the four BTEX compounds are listed in Table 3. The selected 
wavelengths correspond well with the peaks seen in the single-component spectra of the analytes (e.g., Fig.s $10 \mathrm{~A}$ and B).

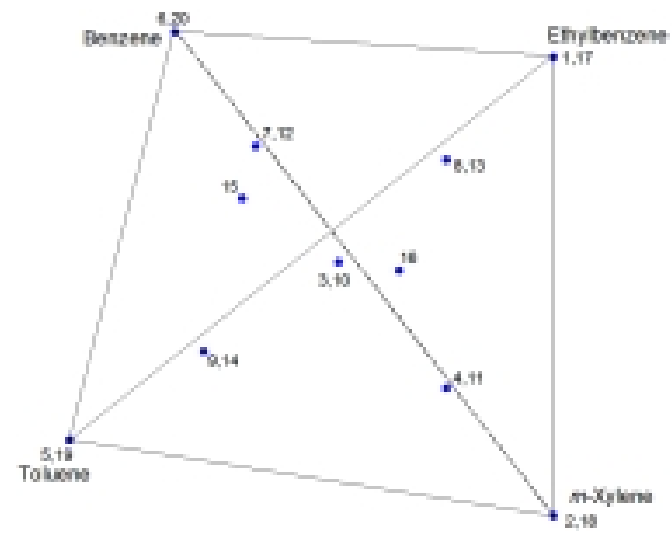

Fig. 8. Simplex mixture design in 4-components.

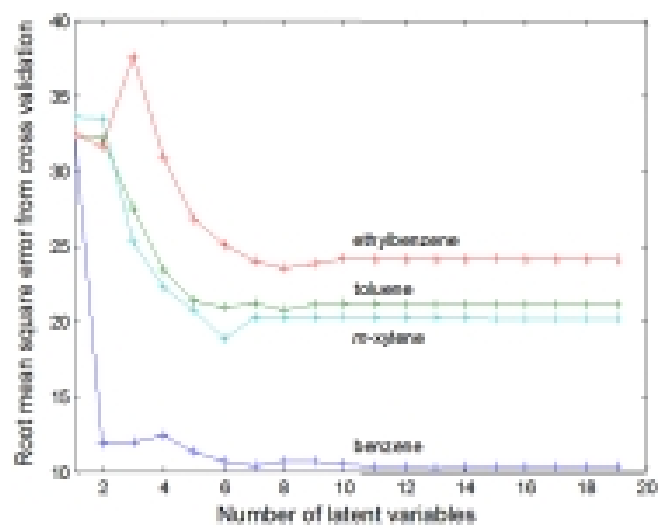

Fig. 9. Root mean square error from cross validation vs. latent variables.

These preliminary data are promising and relevant to the project objectives. This data set was contaminated by extreme noise from OPO excitation source, laser power fluctuations; because of laser fluctuations attempts at calibration using single wavelengths were not successful. Although the cross validated error was relatively high, PLS modeling explicitly modeled the concentration dependence in the presence of the laser power fluctuations. Furthermore, regression coefficients for the simultaneous-analyte PLS model identified wavelengths that were informative for predicting the concentration of the BTEX compounds. These wavelengths matched the location of peaks in the pure component spectra of each compound. Because acquiring excitation scans in the field for wavelength selection can require a great deal of time, the rapid identification of selective wavelengths may be applicable to field analysis.
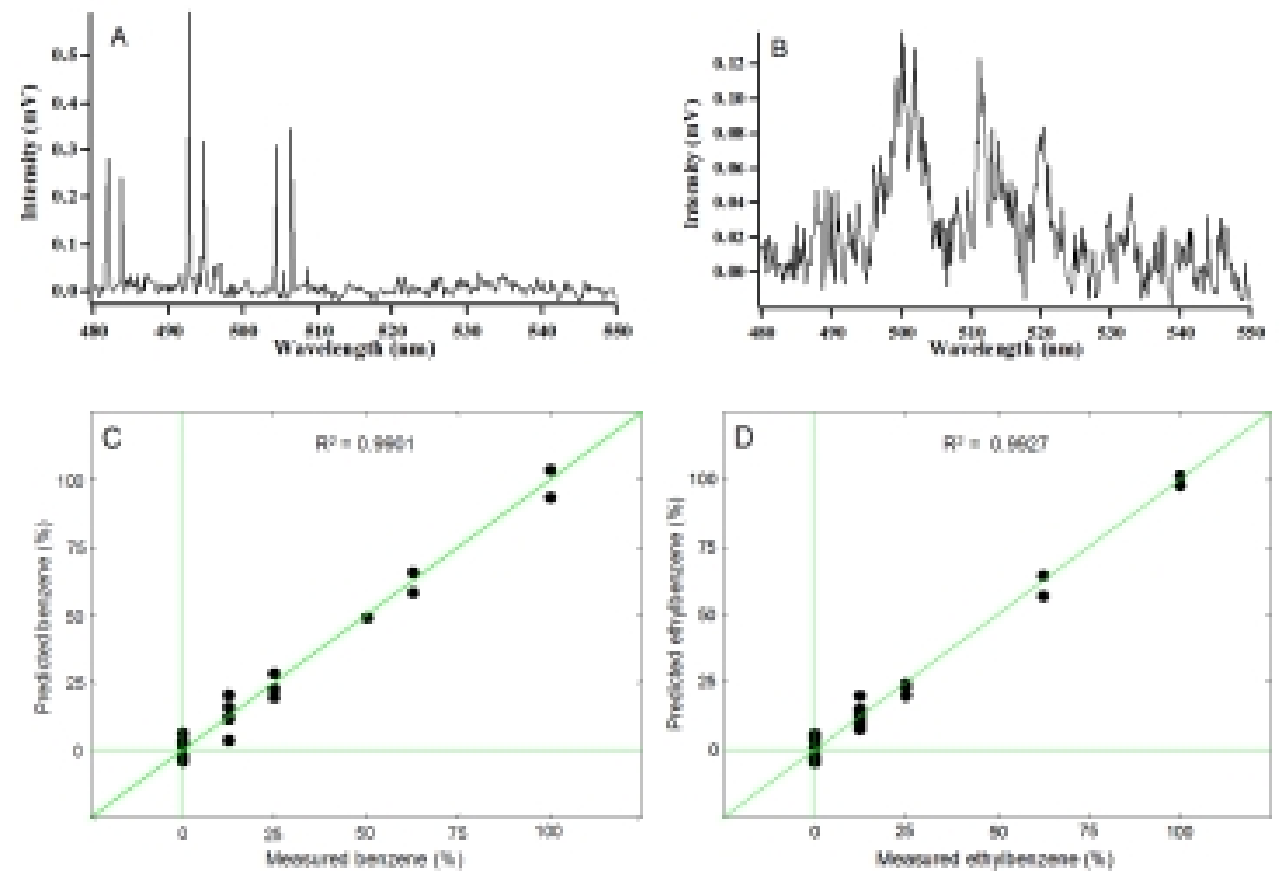

Fig. 10. (A, B) Spectra of benzene and ethylbenzene; (C, D) PLS calibration plots for benzene and ethylbenzene. 
Table 3. Selective excitation wavelengths for BTEX compounds.

\begin{tabular}{lll}
\hline Compound & Variable Number & Wavelength $(\mathrm{nm})$ \\
benzene & $11,20,65,75,133$ & $482.0,483.8,492.8,495.0,506.4$ \\
toluene & $100,103,205,220$ & $499.8,500.4,520.8,523.0$ \\
ethylbenzene & $99,111,157,159,265$ & $500.0,502.0,511.2,511.8,532.8$ \\
$m$-xylene & $132,195,199$ & $506.2,519.0,519.6$
\end{tabular}

Task 4. Soil Column Experiments. We will determine how the laboratory REMPI probe behaves in a laboratory soil column in the presence of water vapor. The soil column (see Fig. 11) was designed and validated by Gribb's group during the prior proposal period and will be constructed at LLNL. A series of soil column experiments to determine the applicability of the proposed fiberoptic REMPI probe to function as a sensor for organic vapors will be performed. The range of organic vapor concentrations for which the sensor must perform varies with the quantity and nature of the chemical in question, soil properties such as soil type, water content, organic carbon content, and environmental conditions such as temperature. In the case of free product and dry soil with no organic carbon, the soil vapor concentration will be at a maximum that can be estimated from Raoult's law. Lower vapor concentrations are expected under most field conditions, depending on the amount of contaminant present and the degree of partitioning of the contaminant between the solid (e.g., sorption to soil solids and/or organic matter), liquid (free product or dissolved phase), and gas phases. Therefore, we will run experiments with one chlorinated solvent, PCE, and one BTEX compound, toluene, present as free product in dry soil, as well as experiments in which these compounds are introduced in a water mixture to soil columns with varying moisture levels (Table 4). The experimental set up and testing program are described in the following sections.

Soil column experiments will be performed in a manner similar to that of Burns and Mayne ${ }^{34}$ in their proof-of-concept study of a cone penetrometer-based BTEX (benzene, toluene, ethylbenzene, xylene) sensor assembly. In this work, triaxial calibration chambers constructed of organic-solvent resistant materials will be used to contain the soil matrix and sensor assembly. The columns will be pyrex glass, the fittings made of stainless steel, and the o-rings made of Vitron. Teflon inflow and outflow tubing will be used for filling and draining the columns with water, TCE, toluene, and TCE/water toluene/water mixtures. The REMPI probe will be enclosed in a stainless steel tube, with a diameter equivalent to that of a standard cone penetrometer $(3.57 \mathrm{~cm})$ and

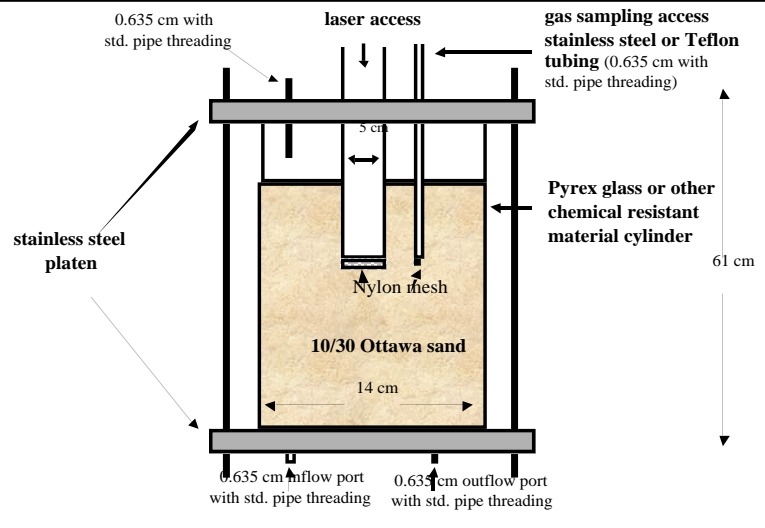

Fig. 11. Soil column design to test water and other interferent effects. remain fixed at a constant depth in the soil during testing. A parallel teflon sampling tube will be adjacent to the REMPI probe housing for gas sampling purposes. Ottawa 20/30 sand will be used as the soil matrix. This well-characterized sand soil is composed of essentially pure silica, which is nonreactive (e.g., no sorption of organics to soil solids will occur).

Known volumes of TCE or toluene will be injected into the columns of dry and partially saturated soil columns. In all cases, the REMPI probe will be above the point where the soil is saturated with the liquid phase. In the first set of experiments, pure solvent will be injected into dry soil from the 
base of the column. As the soil-solvent-air-system comes to equilibrium, simultaneous measurements of the soil-gas concentrations will be taken with the REMPI probe, and compared to gas chromatography results from soil-gas samples taken at the same depth in the soil column. In subsequent experiments, the soil will be saturated with water from the base of the column and then drained to obtain partially saturated soil conditions. Then water/TCE or water/toluene mixtures with varying organic concentrations will be injected into the base of the soil column. In these experiments, the lower portion of the column will remain saturated to different depths, to obtain different average moisture content regimes. This will allow us to approximate different potential field conditions. The test matrix shown in Table 4 will be completed. Sets of tests will be performed in dry, low, medium, and high moisture content soil columns at three different temperatures: 15,20 , and $25^{\circ} \mathrm{C}$. This will allow us to determine the effects of ambient soil moisture content and temperature. Three tests will be run to check the repeatability of the sensor measurements.

Table 4. Test matrix for soil column experiments.

\begin{tabular}{lllll}
\hline Exp. No. & Chemical & Concentration & Soil Matrix Conditions & Temperatures \\
\hline \hline $1-6$ & TCE, toluene & free phase & dry & $15,20,25 \mathrm{C}$ \\
$7-24$ & TCE, toluene & low, med, high & dry & $15,20,25 \mathrm{C}$ \\
$25-42$ & TCE, toluene & low, med, high & $\begin{array}{l}\text { partially saturated, low overall } \\
\text { moisture content }\end{array}$ & $15,20,25 \mathrm{C}$ \\
& TCE, toluene & low, med, high & $\begin{array}{l}\text { partially saturated, medium } \\
\text { overall moisture content }\end{array}$ & $15,20,25 \mathrm{C}$ \\
43-60 & partially saturated, high overall & $15,20,25 \mathrm{C}$ \\
& TCE, toluene & low, med, high & moisture content & \\
\hline
\end{tabular}

This series of experiments will allow us to address a number of questions, such as: How will the porous medium affect uptake of the organics? How long will it take the REMPI probe to react under the different conditions? Will varying moisture content of the soil impact the reliability or sensitivity of the measurements?

Task 5. Refine the fiber-optic REMPI probe to make third generation REMPI sensors. This task will mainly be carried out at LLNL, since that group has designed several successful fiber-optic sensors for cone penetrometers, including a Raman measurement system. However, Angel's group will contibute as this group also has considerable experience modeling fiber-optic spectroscopy probes for Raman, fluorescence, and LIBS measurements. LLNL will model new electrode designs using an electrostatic filed model, and will construct the probes and deliver them to USC for testing.

The signal generated by a fiber-optic REMPI probe can be described by a model that is similar to a single-fiber Raman probe that we have described. ${ }^{42-43}$ For a fiber-optic REMPI probe with a single optical fiber, the total observed REMPI signal $\mathrm{S}_{R}$, will depend on the laser power density at the fiber tip. Fig. 12 shows a coordinate system that we used to develop models of fiber-optic Raman probe signals. This Fig. and coordinate system also apply to a single-fiber REMPI probe except only the excitation fiber needs to be considered. For a single fiber, the laser power density of the illumination cone incident upon the plane at $h$ is described by the function $g(x, y)$. This power density is not uniform but is, instead, determined by the electromagnetic mode distribution within the excitation fiber. Because there are many thousands of separate modes, the laser irradiance distribution is 
difficult to model exactly but has been reasonably approximated by a truncated-wing Gaussian of the form

$$
\begin{aligned}
g(x, y)=g(r(x, y)) & =\frac{1}{g_{h}}\left(1-\frac{r^{4}}{r_{h}^{4}}\right) \exp \left(-8 r^{2} / r_{h}^{2}\right) & & \left(0 \leq r \leq r_{h}\right) \\
& =0 & & \left(r>r_{h}\right)
\end{aligned}
$$

in which $r$ is the distance, within the plane, of the volume element from the center of the illumination circle or ellipse, $r_{h}$ is the radial distance from the center to the edge of the illuminated area at height $h$, and $g_{h}$ is the normalization factor (i.e., total integrated power over cross-sectional area, $A(h)$, at height $h$ ). The form of this was empirically chosen previously for our Raman probe studies to give a good approximation to the experimentally-observed power distribution density and resembles a Gaussian function truncated at the $4 \sigma$ point.

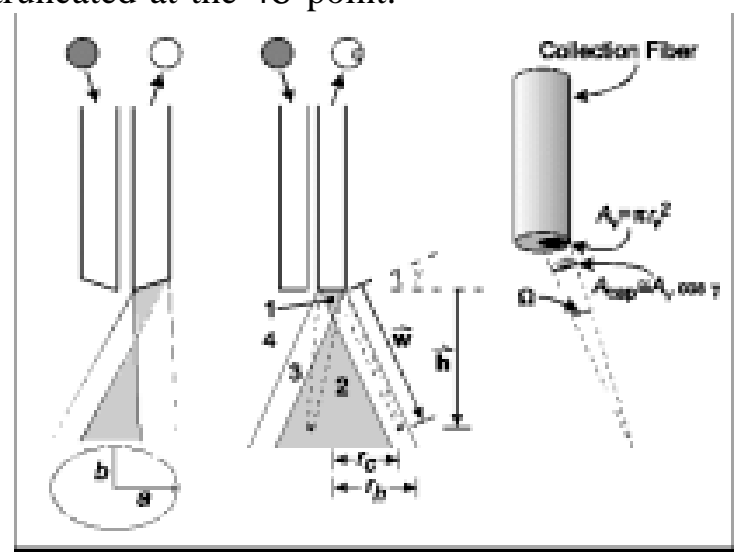

Fig. 12. Diagram of some fiber-optic Raman and fluorescence probes, illustrating the spatial regions corresponding to different definitions of viewing solid angle, $\Omega$, and excitation volume.

In a simplistic approach, the REMPI signal can be found by integrating the product of the power density function and an ionization efficiency function over all $h$. The ionization efficiency function incorporates the laser wavelength and power dependence of the REMPI signal. This assumes $100 \%$ ion collection.

We would like to develop a more quantitative model of the single-fiber REMPI probe. This will require help from LLNL to take into account the efficiency of different electrode configurations. In preliminary work we have calculated the distance dependence of

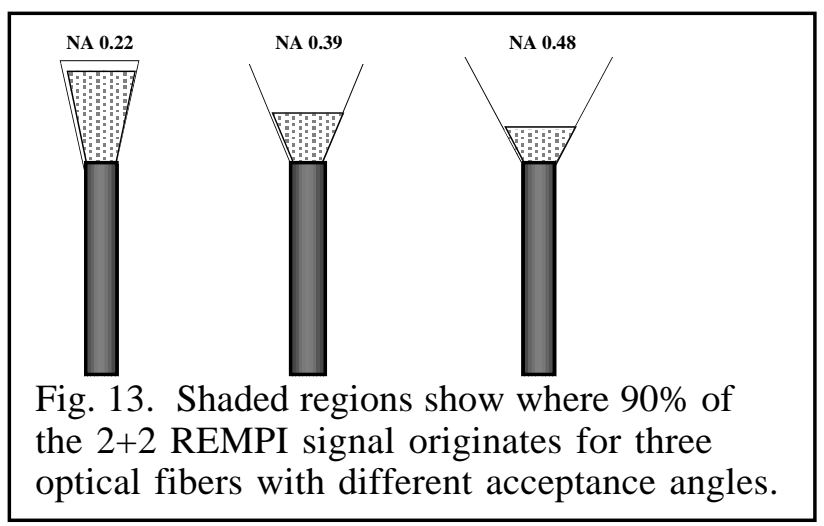
the REMPI signal for different fiber numerical apertures and different excitation schemes. Fig. 13 shows one comparison, the distance at which $90 \%$ of the REMPI occurs for three different fibers assuming 2+2 excitation. Knowledge of this region is important in the optimization of the collection electrode.

Tasks 6 \& 8: Laser desorption studies will be carried out using a system like that shown in Figure 5. We will use a pulsed diode laser as the ablation pulse. This will be compared to the use of a lowcost Nd:YAG laser. Samples to be investigated include VOC, BTEX, Hg and Pb spiked soil samples (these measurements will be made under a well ventilated hood system and metal samples will be 
disposed of according to previously determined USC guidelines). REMPI signal will be monitored as we optimize the desorption pulse energy, and the relative positions of the lasers and electrode.

Task 7: Third generation fiber-optic REMPI sensor using a buffer gas and reduced pressure. In preliminary studies with the sealed REMPI cell we observe much larger signals for all compounds following the introduction of a very small amount of argon gas into the cell. This is shown in Figure 14 for trans-DCE. The REMPI excitation scan around a single DCE peak shows much higher signal when a small amount of Ar gas is introduced. The function of the $\mathrm{Ar}$ is to amplify the ion current and thus increase the sensitivity. As molecular ions are created at low concentrations they continually collide with the easily ionizable $\mathrm{Ar}$ atoms. The result is a much higher ion current and higher sensitivity. We will optimize this effect in terms of amount of Ar and flow rate. This simple technique can be used at atmospheric pressure, and will be incorporated into a fiber-optic REMPI probe. One possible design is shown in Figure 15. Ar gas is introduced using an extremely smalldiameter capillary tube. A similar tube will be used to pull a slight vacuum on the probe to reduce the pressure in the cell, for added sensitivity and selectivity.

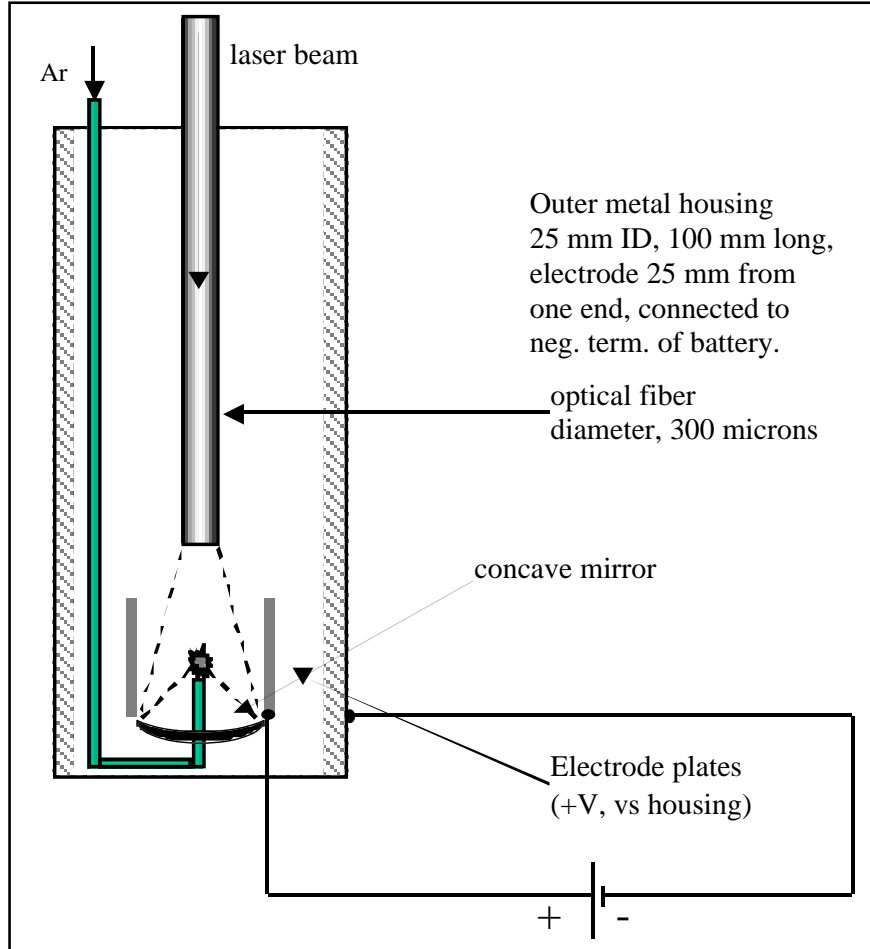

Fig. 15. Third generation probe design using Ar for enhanced signal. 